\title{
Compact Radiative Control Structures for Millimeter Astronomy
}

\author{
Ari D. Brown ${ }^{* a, b}$, David T. Chuss ${ }^{\mathrm{a}}$, James A. Chervenak ${ }^{\mathrm{a}}$, \\ Ross M. Henry ${ }^{a}$, S. Harvey Moseley ${ }^{a}$, Edward J. Wollack ${ }^{a}$ \\ aNASA Goddard Space Flight Center, Greenbelt, MD USA 20771; \\ ${ }^{b}$ MEI Technologies, Inc, 7404 Executive Place, Suite 500, Seabrook, MD USA 20706
}

\begin{abstract}
We have designed, fabricated, and tested compact radiative control structures, including antireflection coatings and resonant absorbers, for millimeter through submillimeter wave astronomy. The antireflection coatings consist of micromachined single crystal silicon dielectric sub-wavelength honeycombs. The effective dielectric constant of the structures is set by the honeycomb cell geometry. The resonant absorbers consist of pieces of solid single crystal silicon substrate and thin phosphorus implanted regions whose sheet resistance is tailored to maximize absorption by the structure. We present an implantation model that can be used to predict the ion energy and dose required for obtaining a target implant layer sheet resistance. A neutral density filter, a hybrid of a silicon dielectric honeycomb with an implanted region, has also been fabricated with this basic approach. These radiative control structures are scalable and compatible for use large focal plane detector arrays.
\end{abstract}

Keywords: Anti-reflection coatings, far-infrared, sub-millimeter, silicon quasioptics, resonant absorbers, neutral density filters

\section{INTRODUCTION}

Recent advances in filled arrays of planar bolometers have driven astronomical discovery in the millimeter through submillimeter portion of the electromagnetic spectrum. Innovative architectures have led to the production of arrays of semiconducting bolometers having hundreds of detectors [1,2]. More recently, advances in transition edge sensors (TES) and corresponding multiplexing technology are enabling a new generation of kilopixel bolometer arrays $[3,4]$.

Typically, it is not practical to scale the architecture in order to maintain the same ratio of pixel size to wavelength in every application. Therefore, the versatility of these detectors depends upon the capability to tailor the radiative coupling strategy to the wavelength of interest. For example, in the submillimeter, control of stray light can be accomplished by broadband lossy structures that are several wavelengths thick [2]. Using the same pixel pitch in the millimeter does not allow such an implementation due to practical physical working volumes limitations. Furthermore, identification of mechanically compatible materials with the required dielectric properties tends to favor a single material solution. It is for these reasons new techniques for anti-reflection coating [5,6], glint reduction, and signal absorption are desirable for long wavelength sensors [7]. In this work we explore several structures that are potential tools for addressing the concerns outlined above. All of these structures are single crystal silicon-based so as to thermally match the detector structures and fabrication processes.

The first structure we consider is a simple resonant absorber with an antireflection coating. In this case, a silicon substrate with an appropriate thickness is implanted with an absorbing conductive layer. The layer impedance is chosen such that the silicon acts as a stepped-impedance antireflection layer for the absorbing layer. This structure has the advantage in that it is an effective absorber over a finite bandwidth and a very thin profile due the use of silicon.

*ari.d.brown@nasa.gov; phone 1301 286-2293; fax 1301 286-1672; 
The second two devices discussed here implement a technique of artificially producing dielectric materials for which the index is lower than that of silicon. This is done by micromachining hexagonal holes through a silicon wafer, which is a controlled method of making porous silicon [8]. If the wavelength of interest is significantly larger than the scale of the hexagonal pore, the medium has an effective dielectric constant that is related to the filling fraction of silicon remaining in the structure. This ability to tailor the effective dielectric constant of a material offers an additional degree of freedom when designing the radiative control structure for a detector system. The first of these fabricated devices is a simple artificial dielectric, which we denote here as "silicon honeycombs". The second was an artificial dielectric with an absorbing layer on one side, which we dub a "hybrid structure" which can serve as a neutral density filter.

\section{BACKGROUND}

\subsection{Effective Medium Theory}

To realize an antireflection (AR) coating from micro-machined silicon we synthesize an artificial dielectric having a dielectric constant that is the geometric mean of the two media one is trying to match. The reflected wave will be cancelled when the thickness $t=\lambda / 4 \varepsilon_{\mathrm{AR}}^{1 / 2}$, where $\lambda$ is the wavelength of the incident radiation and $\varepsilon_{\mathrm{AR}}$ is the dielectric constant of the artificial dielectric. We achieved the desired dielectric constant by controlling the wall thickness $w$ and honeycomb facet dimension $r$ of the silicon layer. See Figure 1. In the low frequency limit (i.e., when the honeycomb pitch $p<<\lambda / 2$ ) the effective dielectric constant can be expressed as a diagonal tensor [9]. Using a numerical technique [10] to obtain the effective dielectric constant for a honeycomb structure, Smith [9] has yielded the following empirical relations for the real part of the effective dielectric tensor for the $\mathrm{xx}$ and yy components:

$$
\begin{aligned}
& \varepsilon_{\mathrm{XX}}=1+w / r\left[0.7375 \varepsilon_{S i}-0.5210\right] \\
& \varepsilon_{\mathrm{YY}}=1+w / r\left[0.6715 \varepsilon_{S i}-0.4615\right],
\end{aligned}
$$

where the dielectric constant of bulk silicon is $\varepsilon_{\mathrm{Si}}=11.7$ [11]. We have applied these generic relations to engineer the optical properties of our silicon AR coatings. We use these relations as a starting point for design procedure. 


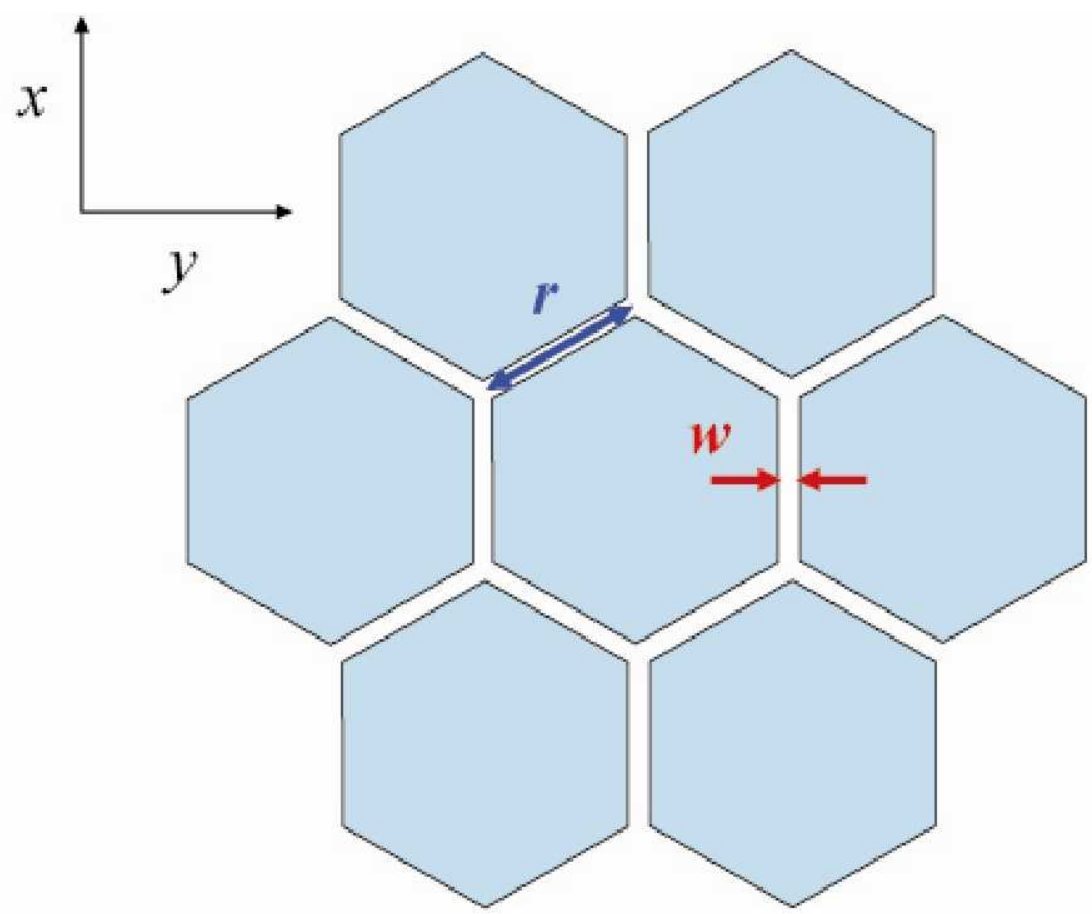

Figure 1. Schematic of a regular honeycomb structure, which made up the basic design of the AR coatings studied here. The shaded regions correspond to pores in the dielectric.

At wavelength below $2 p$ diffraction becomes important and the honeycomb structure can no longer be thought of as a lumped element with a static effective dielectric constant. Consequently, CST Microstripes $^{\mathrm{TM}}$ [12], a commercially available finite difference time domain solver, is used to model the honeycomb structures to study this effect. Selected results from this modeling effort are shown in Figure 2. The solution elucidates the behavior of the honeycomb structure in different wavenumber regimes. The solution compares well with a transmission line model (TLM) calculation in the wavenumber regimes of $0-15 \mathrm{~cm}^{-1}$ and $\sim 60-80 \mathrm{~cm}^{-1}$. In the former regime, the structure can be treated as having a quasistatic effective dielectric constant equal to 3.4. This is in agreement with Smith's predictions. On the other hand, in the latter regime, the numerical solution shows that the behavior structure approaches that of bulk silicon - aside from the occasional resonances arising from radiation being trapped inside the material. In the current study, we are interested primarily in fabricating quasi-optical structures for wavelength $\sim 2 \mathrm{~mm}\left(\sim 5 \mathrm{~cm}^{-1}\right)$. Consequently, a TLM of a quasistatic dielectric is sufficient for adequately describing our structures for our purposes. 


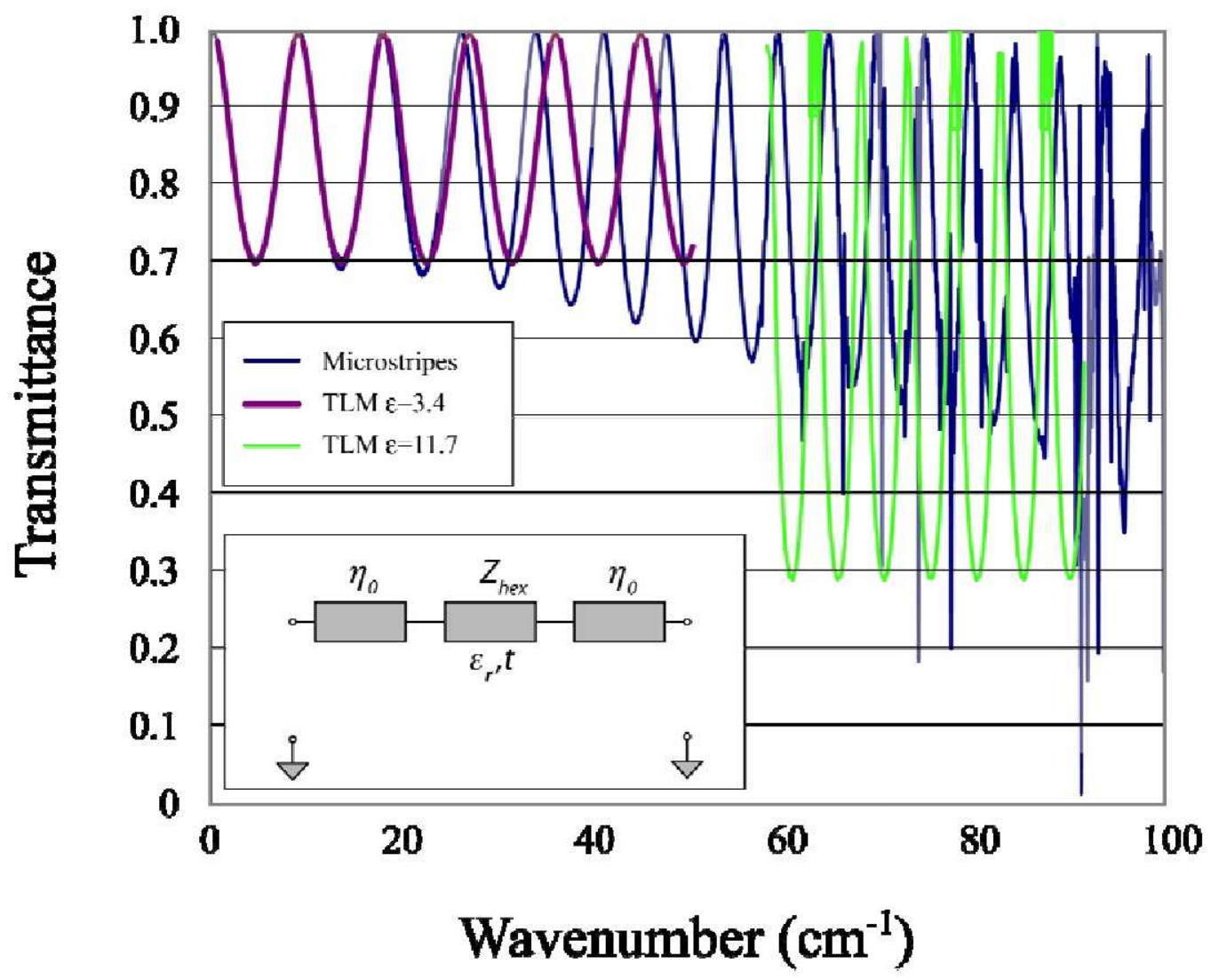

Figure 2. The result of a full wave analysis of the silicon hex structure described in the text is shown (solid line). The dashed lines represent the transmission line model response for dielectric constants of 3.42 and 11.7, respectively. These are the long wavelength and bulk material limits for the silicon structure.

\subsection{Implantation Model}

This artificial dielectric can also be used to enhance the absorption of a free standing silicon layer. In order for the resonant absorber to effectively capture radiation incident on a focal plane detector array, the antireflection conditions, $t$ $=\lambda / 4 \varepsilon_{\mathrm{Si}^{1}}$, must be satisfied. Given that $\varepsilon_{\mathrm{Si}}=11.7$ and our target $\lambda_{0}=2 \mathrm{~mm}$, the absorber thickness has to equal $146 \mu \mathrm{m}$. Furthermore, the target sheet resistance of the implant $R_{\mathrm{abs}}=\eta_{0} / \varepsilon_{\mathrm{Si}} \sim 32.2 \Omega$ per square, where $\eta_{0}$ is the impedance of free space. A transmission line model [13] was used to predict the response for this configuration and is shown in Figure 3. 


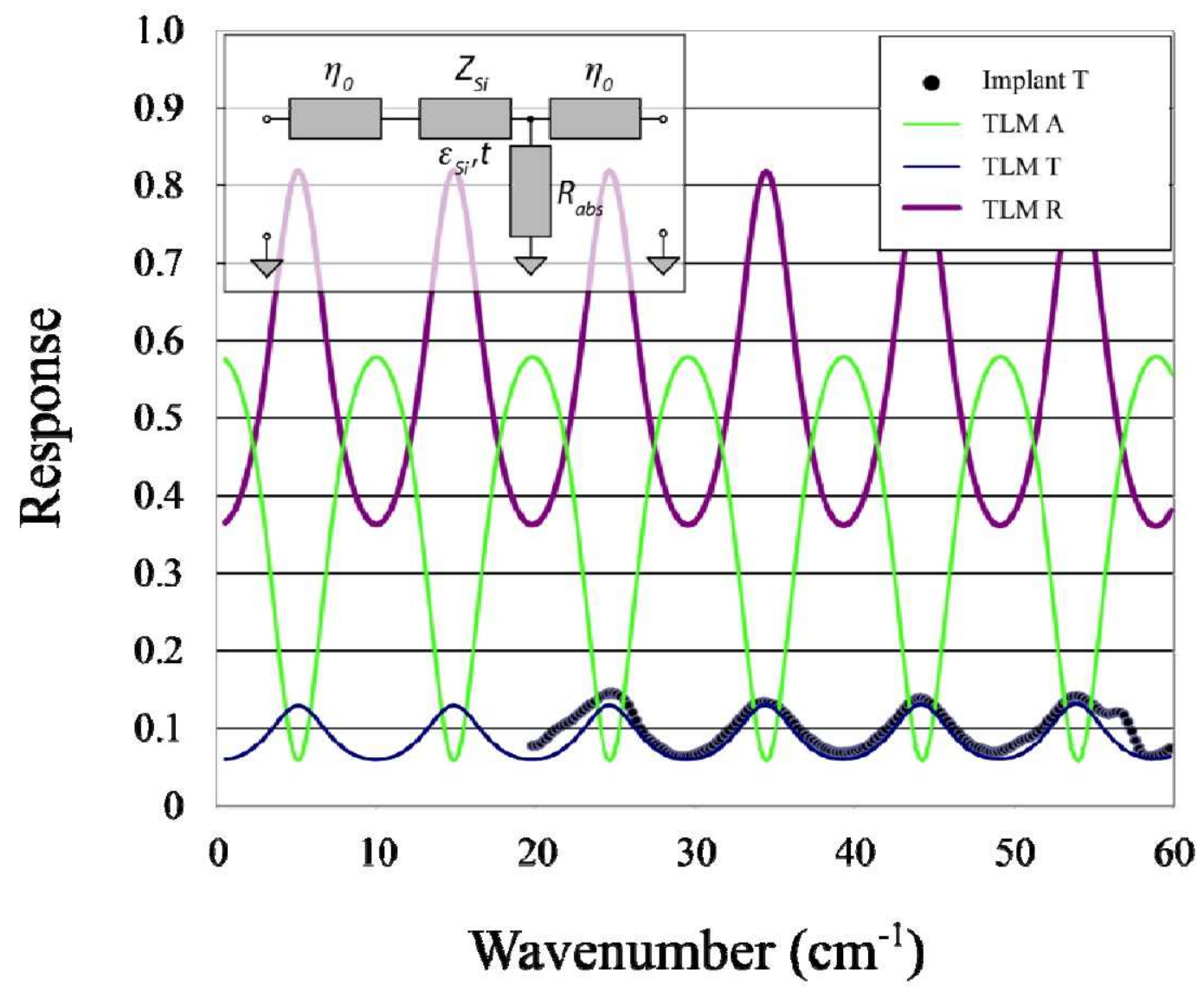

Figure 3. Simulated optical response of a resonant absorber, which consists of 146 micron thick bulk silicon layer with a $32.2 \mathrm{Ohm}$ per square implant on the backside of the substrate. Transmission data for an implanted Si sample are also shown.

We constructed an implantation model in order to guide our fabrication methodology so as to achieve a desired sheet resistance. First, we interpolated phosphorus ion range and straggle values in silicon for a given ion energy by using data tabulated by Brice [14]. The 1-D concentration profile of implanted phosphorus was assumed to have a Gaussian distribution:

$$
c(z)=c_{0}+\frac{\Phi}{\sqrt{2 \pi} s} e^{-\left[(z-r)^{2} / 2 s^{2}\right]}
$$

where $c_{\mathrm{o}}$ is initial implant concentration, $\phi$ is the ion dose per unit area, $s$ is the ion straggle, $r$ is the ion range, and $z$ is the distance from the substrate surface. In order to account for implant diffusion in the Si bulk during thermal activation by solving Fick's second law:

$$
\frac{d c}{d t}=D \frac{d^{2} c}{d z^{2}}+\frac{d D}{d z} \frac{d c}{d z}
$$

The phosphorus diffusivity $D$ in silicon at high phosphorus concentration was assumed to be 


$$
D(c(z), z)=D_{1}+D_{2} \frac{c(z)}{c_{i}}+D_{3}\left(\frac{c(z)}{c_{i}}\right)^{2},
$$

where $D_{1}$ represents the diffusivity of interstitials, $D_{2}$ and $D_{3}$ represent diffusivity of vacancies and divacancies, respectively, and $c_{\mathrm{i}}$ is the intrinsic carrier concentration [15]. Complexities associated with phosphorus diffusion at the silicon surface were eliminated, because a silicon dioxide etch stop was grown on top of the silicon prior to implantation. In order to account for the influence of the oxide layer, a corrected value for the $\mathrm{P}^{+}$ion range was used in our calculations. We then integrated Fick's second law numerically using a forward in time centered in space algorithm [16] to obtain a post-annealing activated phosphorus concentration. The sheet resistance of the absorber,

$$
R_{a b s}=\left(e \int c(z) m(z) d z\right)^{-1},
$$

in which $m(z)$ is the electron mobility. The exact form of mobility used in our model can be found in Reference 17.

The modeled phosphorus concentration distribution prior to and after activation for a parameter set (e.g., ion energy and dose, and oxide thickness) used in fabrication is depicted in Figure 4. The model predicts that the concentration distribution is much more sharply peaked prior to activation than after activation. Nonetheless, even after activation, $95 \%$ of the implant is contained within a silicon near-surface region that is only $170 \mathrm{~nm}$ thick. This is very small as compared to the 10-100 microns of the silicon wafers used to fabricate AR coatings and resonant absorbers. Furthermore, we can treat the implanted layer as being thin compared to the electrical skin depth, which is approximately one micron for the wavelength range of interest.

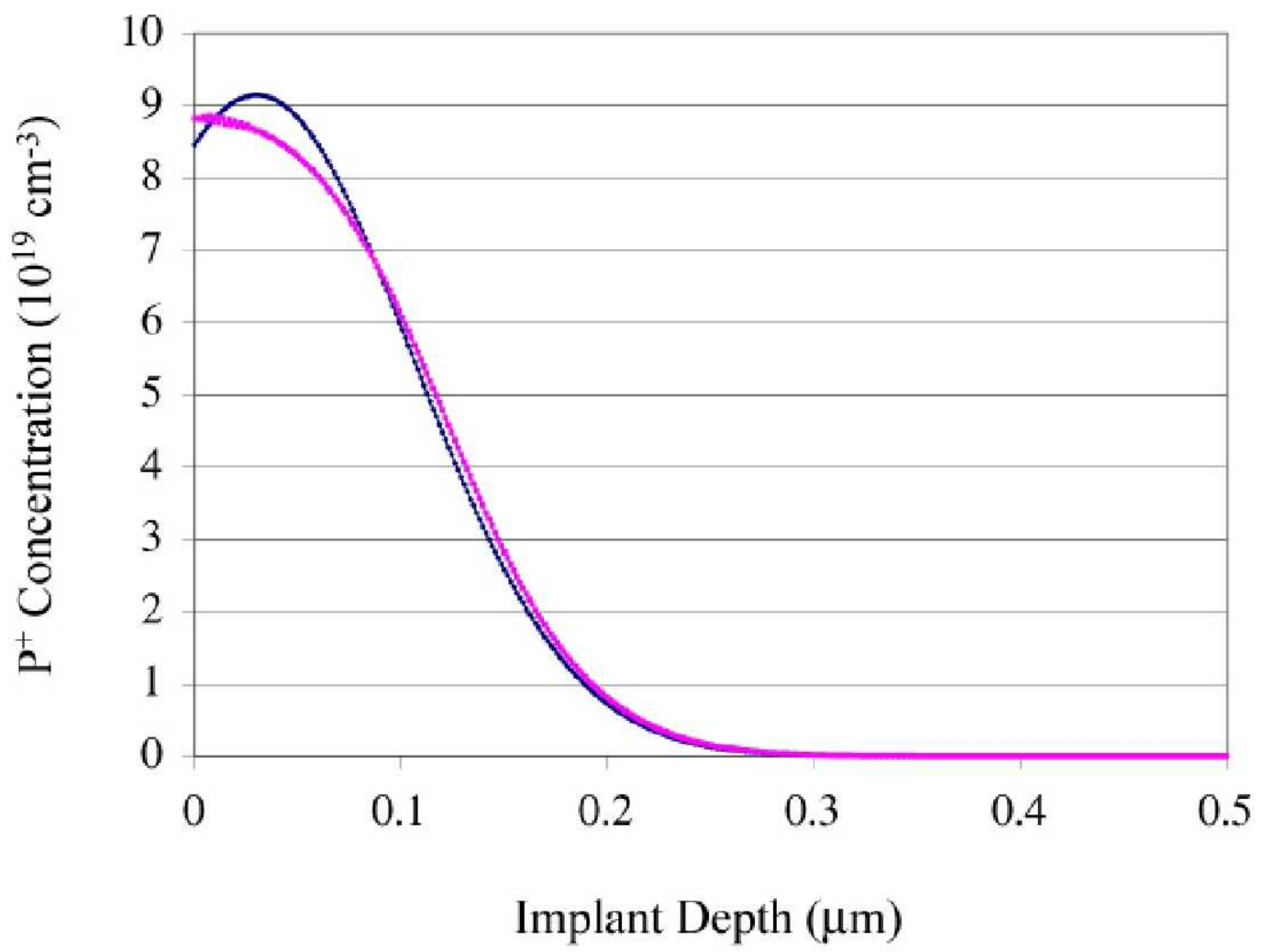

Figure 4. Modeled implanted phosphorus concentrations prior to and after activation (blue and pink curves, respectively). The $\mathrm{P}^{+}$ion energy and dose were $190 \mathrm{keV}$ and $1.73 \times 10^{15} \mathrm{~cm}^{-2}$, respectively, and the $\mathrm{SiO}_{2}$ diffusion barrier layer was $210.0 \mathrm{~nm}$ thick. The estimated sheet resistance post-activation is $30.0 \mathrm{Ohms} / \mathrm{sq}$ at $4.2 \mathrm{~K}$. 


\section{FABRICATION}

Silicon dielectric honeycombs were fabricated inside of a class 100 cleanroom using standard semiconductor processing techniques. Four inch $\mathrm{Si}(001)$ device wafers $(\mathrm{Fz}, \mathrm{n}$-type, $\rho=10-15 \mathrm{k} \Omega \mathrm{cm})$ were bonded to 4" Si backing wafers using Crystalbond-509/509S wax. The device wafers were then patterned via photolithography using AZ-4620 photoresist. Subsequently, we etched the silicon in a deep reactive ion etcher (DRIE) using the Bosch process, in which an $\mathrm{SF}_{6}{ }^{+} / \mathrm{O}_{2}{ }^{+}$ plasma was used to etch and a $\mathrm{C}_{4} \mathrm{~F}_{8}{ }^{+}$plasma was used to passivate the silicon surface. Passivation is required in order to prevent feature undercutting, and we have been able to modify our DRIE recipes so as to obtain very steep sidewall profiles. It is necessary that the sidewall profiles be steep so that accurate modeling of the optical properties of the antireflective coating can be conducted. In order to prevent wax from being resputtered onto the device wafer during DRIE processing, a thermally grown $\sim 400 \mathrm{~nm}$ thick $\mathrm{SiO}_{2}$ layer was deposited on the backside prior to wax bonding and served as an etch stop. The antireflection coatings were diced and released in acetone. A 1:10 $\mathrm{HF}: \mathrm{H}_{2} \mathrm{O}$ solution was used to strip $\mathrm{SiO}_{2}$ from the backside of the devices, which consisted of honeycomb arrays extending over a $1 \mathrm{~cm} \mathrm{x} 1 \mathrm{~cm}$ area.

Figure 5 shows representative scanning electron microscope (SEM) micrographs of the top (Fig. 5a), i.e., facing the DRIE plasma, and bottom (Fig. 5b) faces of silicon honeycomb arrays, in which our target, which was patterned in photoresist, $w, r$, and $p$ were $13.6,40.4$, and 70 microns, respectively. These values are predicted to yield $\varepsilon=3.6$, which was expected to drop to approximately $\sim 3.4$ when reductions in $w$, due to slight photoresist removal [18] during oxygen plasma cleaning prior to DRIE etching, were accounted for. In both cases, the measured $w$ is smaller than the features in the photolithography mask. This observation suggests that the photoresist was undercut during development. We observe that the topside of the silicon surface remains unharmed during reactive ion etching, which indicates that the AZ-4620 photoresist is effective as a passivation layer. On the other hand, we find that by taking the difference in $w$ between the top and bottom faces the bottom face of the honeycomb is partially undercut. We believe that this may be attributed to non-uniformities (typically 5-10\%) in the DRIE etching rate. The outer regions on the wafer are completed cleared of silicon much more quickly than the central region. This is because some regions on the wafer will be etched all the way through much more quickly than others. Consequently, charge builds up in the silicon dioxide layer abutting these high silicon etch rate regions. This causes $\mathrm{SF}_{6}{ }^{+}$ions to be deflected, some of which contribute to sidewall etching. We have managed to modify our DRIE recipes in order to minimize the magnitude of this effect, which is typically three microns of undercut for a 300 micron trench. The degree of sidewall sharpness can be seen in Figure 5c. It is also important to note that the sidewall roughness is very small. Figure $5 \mathrm{~d}$ reveals that the sidewall roughness is of order one micron or less. 


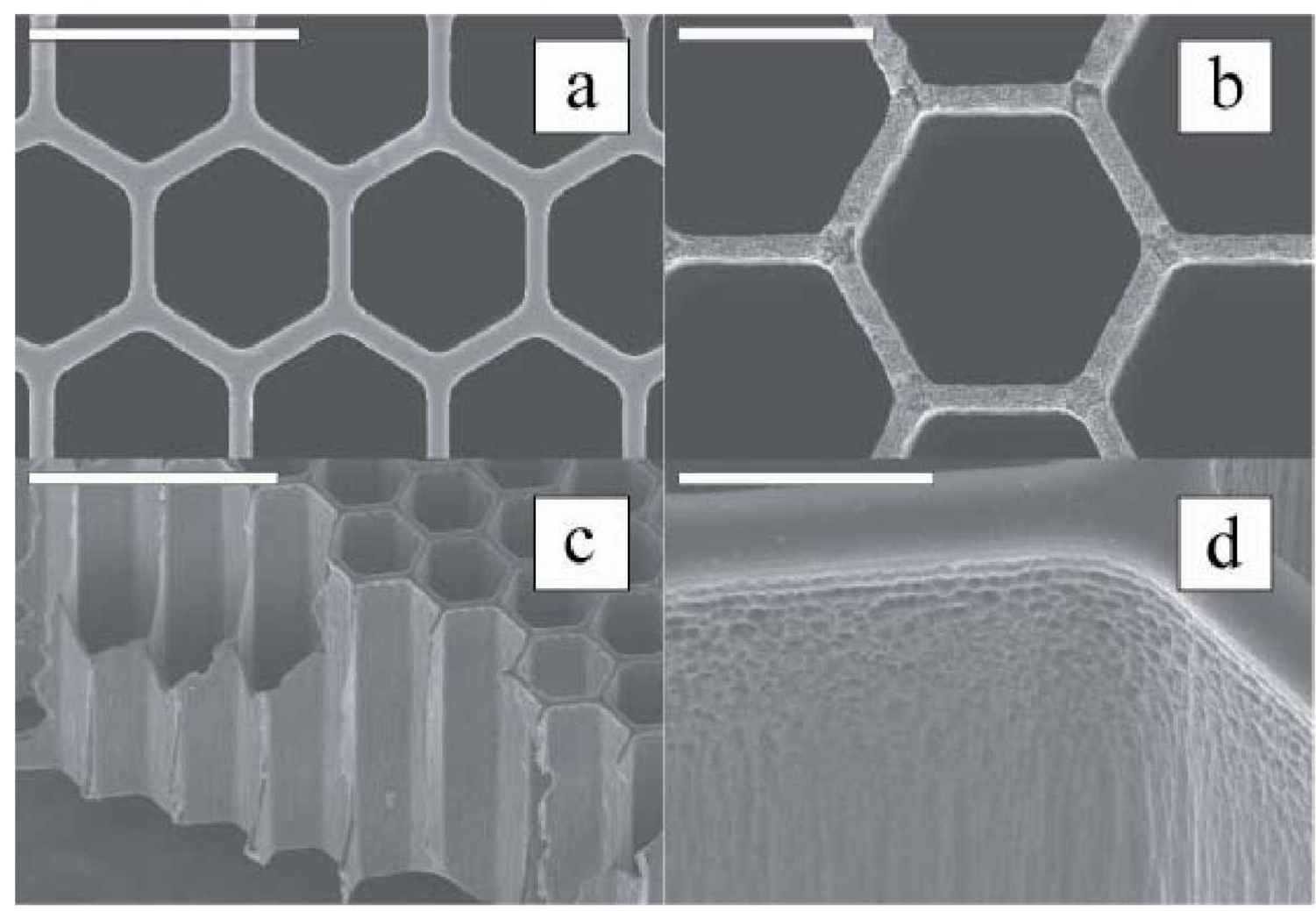

Figure 5. SEM micrographs of DRIE silicon honeycombs. (a) Plan view of the topside of the silicon surface, $w \sim$ $6.5 \pm 1$ microns. The surface is pristine, indicating that the surface was effectively passivated by the photoresist. (b) Plan view of the bottom side of the silicon surface; undercutting is evident, as we measure $w \sim 2.5 \pm 1$ microns. (c), (d) Side views of the honeycomb structure. (d) shows that the sidewall is slightly tapered and is relatively smooth. Scale bar (a) 100 microns, (b) 50 microns, (c) 200 microns, (d) 20 microns.

Silicon absorbers were fabricated out of 4" $\operatorname{Si}(001)$ wafers (Fz, n-type, $\rho=10-15 \mathrm{k} \Omega \mathrm{cm}$ ) that were mechanically thinned to 146 microns and cleaned using a modified RCA process [18]. A $210 \mathrm{~nm}$ thick $\mathrm{SiO}_{2}$ diffusion barrier layer was subsequently thermally deposited inside of a pure, dry oxygen environment. A 400 micron thick silicon wafer was wax bonded to the device wafer prior to further processing in order to prevent to serve as a mechanical support during fabrication. One side of the device wafers were implanted with $1.73 \times 10^{15} \mathrm{~cm}^{-2} 190 \mathrm{keV} \mathrm{P}$ ions directed $7^{\circ}$ from normal, to prevent ion channeling, by Core Systems (Sunnyvale, CA). The device wafers were subsequently released from the backing wafers in acetone, and the implant was activated by annealing the wafers at $900{ }^{\circ} \mathrm{C}$ for 30 minutes in a tube furnace with dry $\mathrm{N}_{2}$ flowing. A 1:10 HF: $\mathrm{H}_{2} \mathrm{O}$ solution was used to strip $\mathrm{SiO}_{2}$ from the devices.

The hybrid structures, which were comprised of a silicon honeycomb and resonant a absorber were fabricated by micromachining a silicon wafer that had first been implanted on one side.

\section{SAMPLE CHARACTERIZATION}

\subsection{Measurement apparatus}

To measure the optical response of the dielectric honeycombs and resonant absorbers a Bruker IFS-113v FTS Genzel interferometer was used to collect data between 2 and 1000 microns. The interferometer was used with a mercury arc source, $50 \mu \mathrm{m}$ mylar beamsplitter, and an Infrared Labs bolometer to gather data in the FIR from roughly $20 \mathrm{~cm}^{-1}-100$ $\mathrm{cm}^{-1}(500 \mu \mathrm{m}-100 \mu \mathrm{m})$. The FTS was then reconfigured to use the same source and detector, but with a $12 \mu \mathrm{m}$ mylar 
beamsplitters which allowed the instrument to gather data between $80 \mathrm{~cm}^{-1}-250 \mathrm{~cm}^{-1}(125 \mu \mathrm{m}-40 \mu \mathrm{m})$. Finally, a 3.5 $\mu \mathrm{m}$ mylar beamsplitter was used to gather data from $200 \mathrm{~cm}^{-1}-650 \mathrm{~cm}^{-1}(50 \mu \mathrm{m}-15 \mu \mathrm{m})$. These different combinations of beamsplitters with the accompanying mercury arc source and bolometer detector allowed the instrument to gather spectral data over the full wavelength range of interest.

Each sample was mounted into a horizontal, two position translation stage. An empty "reference" scan was taken for the particular wavelength region. The sample was then moved into the beam path and a "sample" spectrum was taken. A ratio of the two spectra was taken, which resulted in a spectral transmittance measurement of the sample. This process was repeated with a comparison of the sample to a gold flat to determine the reflectance. The absorption is computed from, $A=1-T-R$, where $T$ and $R$ are the measured sample transmittance and reflectance respectively.

\subsection{Dielectric honeycomb characterization}

The measured dielectric honeycomb transmittance $T$, an example of which is shown in Figure 6, was compared to theory [20]. We made the assumption that the honeycomb structure could be treated as a dielectric of uniform effective dielectric constant and thickness. This form of $T$ compares favorably with the data when $\varepsilon_{\text {eff }}=1.95$ over one interference fringe. In order to obtain a much better fit to the data, we took into account the tapered geometry of the honeycomb walls because of DRIE undercutting. For the fit shown in Fig. 5 we assumed that,

$$
\begin{aligned}
& \varepsilon=\varepsilon_{\text {eff }} ; x \in\left[0, t^{\prime}\right) \\
& \varepsilon=\varepsilon_{\text {eff }}\left[1-\left|\frac{x}{x^{\prime}}\right|\left(\frac{\varepsilon_{\text {eff }}-\varepsilon_{\text {min }}}{\varepsilon_{\text {eff }}}\right)\right] ; x \in\left[t^{\prime}, t\right],
\end{aligned}
$$

where $\varepsilon_{\min }$ is the effective dielectric constant of the higher porosity structure (see Fig. 4(b)) at the bottom of the DRIE trench and $t=t^{\prime}+x$ ' is thickness in which $t^{\prime}$ is the thickness of the "untapered" region. In this case, a best fit was obtained when $\varepsilon_{\mathrm{eff}}=2.30, \varepsilon_{\min }=1.15$, and $x^{\prime}=30$ microns. We note that these extracted values of the effective dielectric constant are almost identical to those predicted from the geometry of the structure (see Fig 5) [9], in which $\varepsilon_{\text {eff }} \sim 2.23$ and 1.44 for the top (Fig. 5a) and bottom (Fig. 5b) surfaces, respectively. 


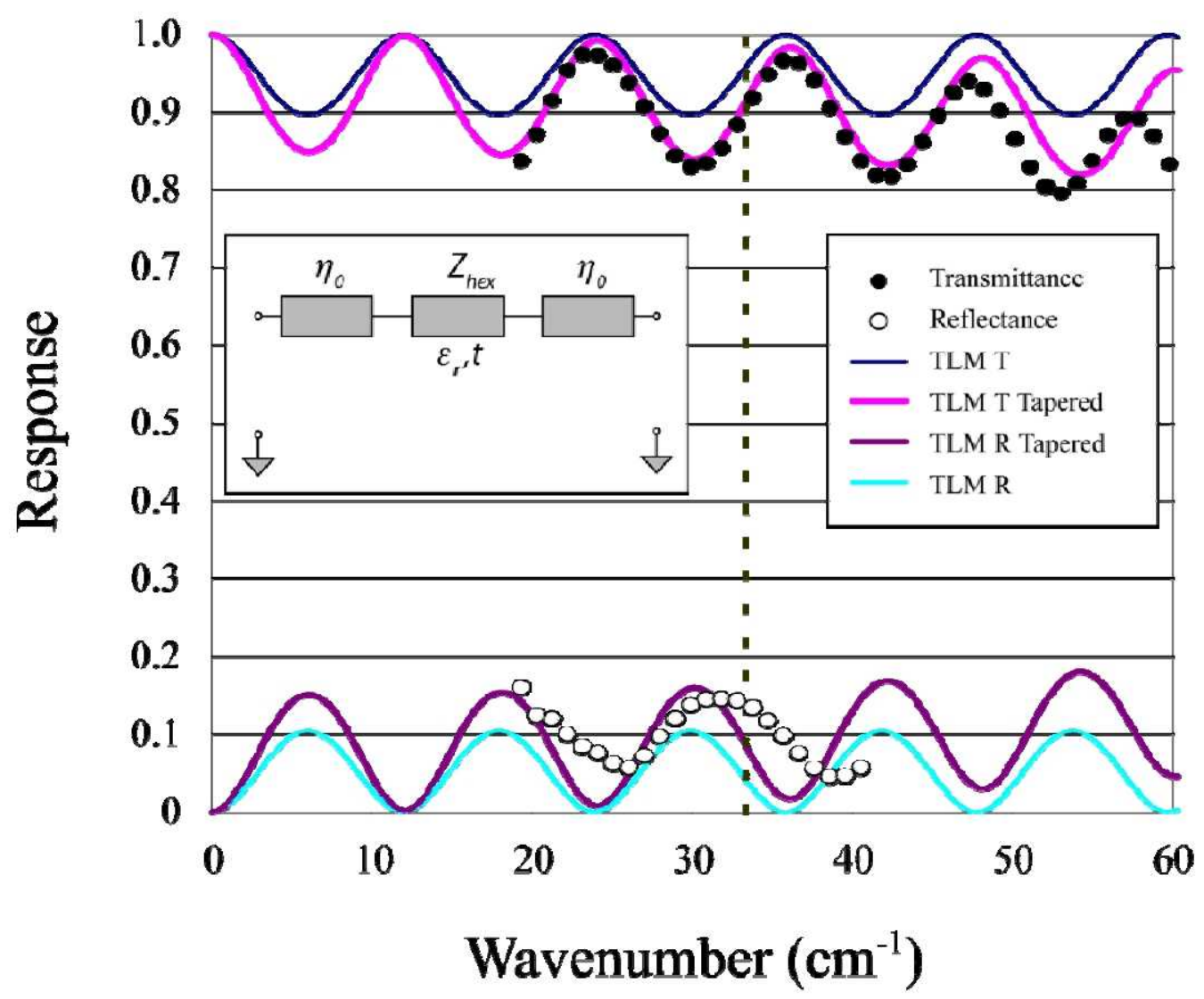

Figure 6. Measured transmittance and reflectance (filled and open circles, respectively) of a free standing 300 micron thick silicon dielectric honeycomb sample. Also shown are TLM values for both quantities, under the assumption that the honeycombs are either uniform or tapered. We obtain a best fit to the transmittance data when $\varepsilon_{\text {eff }}=1.95$ for the uniform case, and $\varepsilon_{\text {eff }}=2.30, \varepsilon_{\min }=1.15$, and $x^{\prime}=30$ microns for the tapered case. The accuracy of the reflectivity data are limited by scattering and reprocessing of light by the FTS in the measurement configuration used. The dashed vertical line indicates the frequency where the structure becomes multi-moded due to diffraction.

\subsection{Dielectric honeycomb with backside absorber characterization}

The complexities associated with handling and processing 140-150 micron thick 4" silicon wafers render high yield resonant absorber fabrication difficult. Thus, in order to minimize these challenges and given our ability to tune the dielectric constant of a silicon wafer via micromachining, we fabricated a hybrid structure which consisted of (1) a resonant backside absorber consisting of 300 micron thick Si honeycomb structure, and (2) an activated phosphorus implant layer. A resonant absorber for $2 \mathrm{~mm}$ radiation can be accomplished by tuning the dielectric constant of the silicon honeycomb to $\varepsilon_{\mathrm{hex}}=3.42$ and the sheet resistance of the absorber to $R_{\mathrm{abs}}=\eta_{0} f / \varepsilon_{\mathrm{Si}}{ }^{1 / 2}$, where $f$ is the honeycomb filling fraction. We fabricated a honeycomb structure with $w=12.6$ microns and $r=40.4$ microns which was required to obtain a silicon honeycomb dielectric constant of 3.42. The filling fraction of this structure was 0.347 and, consequently, the target sheet resistance for this structure was $38.3 \Omega / \square$.

FTS measurements (Fig. 7) show that for $15-75 \mathrm{~cm}^{-1}$ the free standing structure serves as a neutral density filter. We use a transmission line model which assumes a filling fraction to extract the effective absorber sheet impedance of $R_{\text {abs }} / f \sim$ $110 \mathrm{Ohms}$ per square. This realizes the frequency independent absorber condition of [21]. We also use the structure's geometry and material properties in Microstripes to model derive the sheet impedance of the implant from the FTS data. Both methods indicate the effective implant sheet resistance, $~ 38 \mathrm{Ohms}$ per square, was achieved in fabrication. An 
optical of a witness sample consisting of solid piece of implanted silicon taken from the same wafer is in agreement with these results.

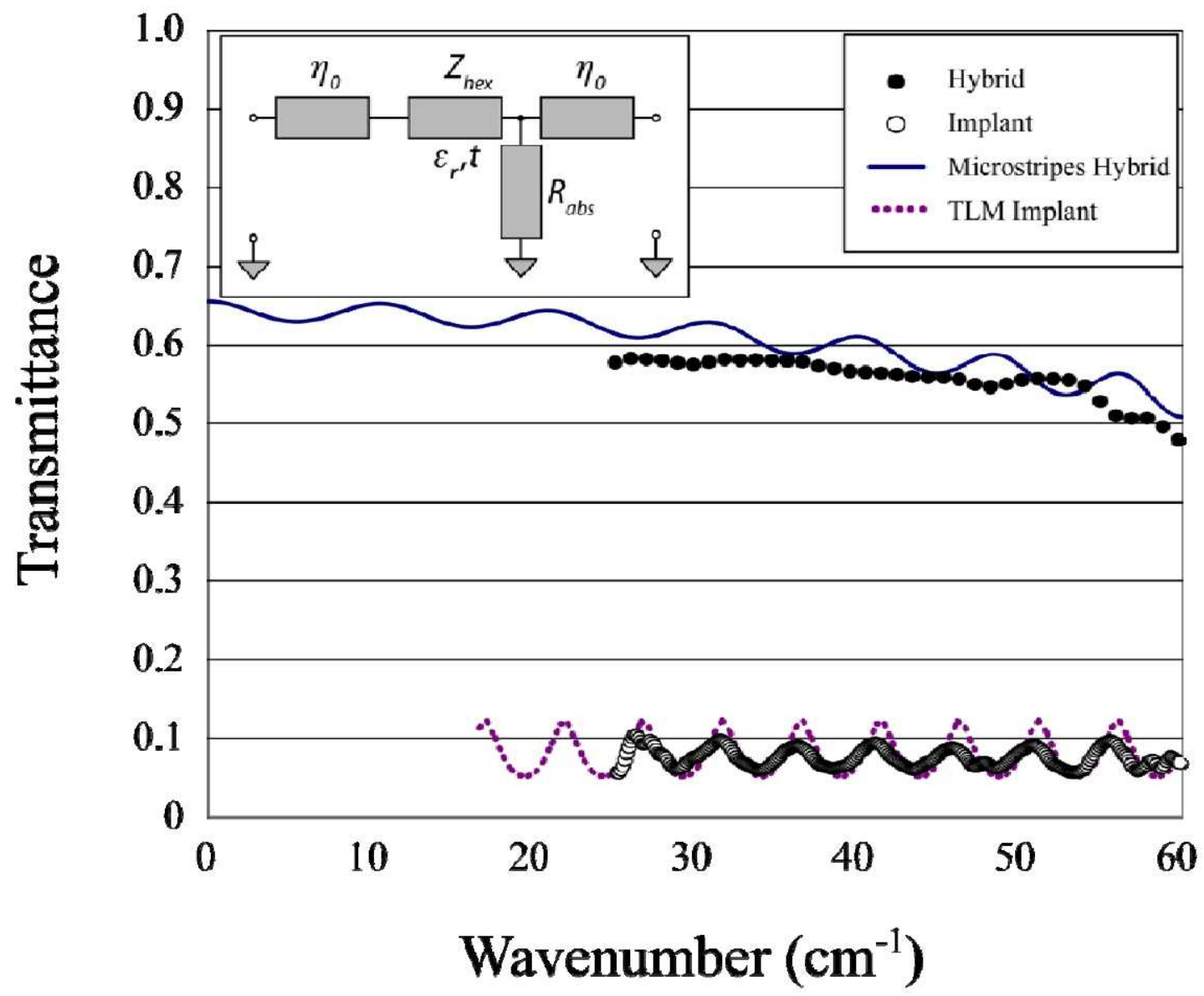

Figure 7. Modeled and measured hybrid structure response. The Microstripes model has a 38.3 implant and an idealized honeycomb tiling in which $w=12.6$ microns and $p=70$ microns (model - solid line; measurement filled circles). Also shown are data from an implanted but unpatterned witness sample on the same wafer (model dashed line; measurement - open circles).

\section{CONCLUSIONS}

We present straightforward techniques for fabricating single crystal silicon quasi-optical structures, which include dielectric honeycomb AR coatings and resonant absorbers, for millimeter astronomy. Various models are used to engineer the optical properties of our structures, as these structures can be tailored to operate in different wavelength regimes by varying their geometry and implantation parameters. The range over which these parameters can be varied is large, primarily because of the maturity of the silicon-based semiconductor processing industry. In addition, novel selfassembly fabrication approaches (e.g., see for instance see [22]) can extend this range even further to enhance the versatility of the approach described here.

\section{ACKNOWLEDGEMENTS}

We gratefully thank Thomas Stevenson for useful discussions. This research was supported in part by an appointment (A. D. Brown) to the NASA Postdoctoral Program at NASA Goddard Space Flight Center administered Oak Ridge Associated Universities through a contract with NASA. Funding for this work was provided by NASA Goddard Space Flight Center's 2006 Director's Discretionary Fund. 


\section{REFERENCES}

[1] C. Darren Dowell, C. A. Allen, S. Babu, M. M. Freund, M. B. Gardner, J. Groseth, M. Jhabvala, A. Kovacs, D. C. Lis, S. Harvey Moseley, Jr., T. G. Phillips, R. Silverberg, G. Voellmer, H. Yoshida, "SHARC II: a Caltech Submillimeter Observatory facility camera with 384 pixels,"Proc. SPIE 4855, 73 (2003).

[2] D.A. Harper, C. Allen, M. Amato, T. Ames, A. Bartels, S. Casey, R. Derro, Rh. Evans, I. Gatley, S. Heimsath, A. Hermida, M. Jhabvala, J. Kastner, R. F. Loewenstein, S. H. Moseley, R. J. Pernic, T. Rennick, H. Rhody, D. Sandford, R. Shafer, P. Shirron, G. Voellmer, S. Wang, and J. Wirth, "HAWC: a far-infrared camera for SOFIA," Proc. SPIE 4014, 43 (2000).

[3] J. W. Fowler, "The Atacama Cosmology Telescope," in Millimeter and Submillimeter Detectors for Astronomy II, J. Zmuidzinas, W. S. Holland, and S. Withington, eds., Proc. SPIE 5418, 1 (2004).

[4] W. S. Holland, W. D. Duncan, B. D. Kelley, K. D. Irvin, A. J. Walton, P. A. R. Ade, and E. I. Robson, "SCUBA-2: a large format submillimeter camera on the James Clerk Maxwell Telescope," Proc. SPIE 4855, 1 (2003).

[5] J. Lau, J. Fowler, T. Marriage, L. Page, J. Leong, E. Wishnow, R. Henry, E. Wollack, M. Halpern, D. Marsden, and G. Marsden, "Millimeter-wave antireflection coating for cryogenic silicon lenses," Appl. Optics 45, 2746 (2006).

[6] J. Zhang, P.A.R. Ade, P. Mauskopf, L. Moncelsi, G. Savini, and N. Whitehouse, "New artificial dielectric metamaterial and its application as a terahertz antireflection coating," Appl. Optics 48, 6635 (2009).

[7] E. J. Wollack, D.T. Chuss, and S.H. Moseley, "Electromagnetic considerations for pixellated planar bolometer arrays in the single-mode limit," SPIE Proc. 6275, 62750V (2006).

[8] R. R. Bilyalov, L. Stalmans, L. Schirone, and C. Levy-Clement, "Use of porous silicon antireflection coating in multicrystalline silicon solar cell processing," IEEE Trans. Elec. Dev. 46, 2035 (1999).

[9] F. C. Smith, "Effective permittivity of dielectric honeycombs," IEEE Proc. -Microw. Antennas Propag. 146, 55 (1999).

[10]K. S. Yee, "Numerical solution of initial boundary value problems involving Maxwell's equations in isotropic media," IEEE Trans. Ant. Prop. 14, 302 (1966).

[11]J. W. Lamb, "Miscellaneous data on materials for millimetre and submillimetre optics," Int. J. Infrared Millim. Waves 17, 1997 (1996).

[12] www.cst.com/Content/Products/MST/Overview.aspx

[13] P. F. Goldsmith, [Quasioptical Systems], IEEE Press, New York, (1998).

[14]D. K. Brice, [Ion Implantation Range and Energy Deposition Distributions], Plenum Press, New York, (1975).

[15] S. K. Ghandi, [VLSI Fabrication Principles: Silicon and Gallium Arsenide, $2^{\text {nd }}$ Edition], John Wiley \& Sons, New York, (1994).

[16] W. H. Press, S. A. Teukolsky, W. T. Vetterling, and B. P. Flannery, [Numerical Recipes in C: The Art of Scientific Computing, $2^{\text {nd }}$ Edition], Cambridge University Press, Cambridge, (1992).

[17] G. Baccarani and P. Ostoja, "Electron mobility empirically related to the phosphorus concentration in silicon," Solid-State Elec. 18, 579 (1975).

[18] The sidewall angle of thick ( 13 micron) photoresist that we use is less than $90^{\circ}$. Thus, the bottom edge of the photoresist is susceptible to being cleared away during $\mathrm{O}_{2}{ }^{+}$plasma cleaning. Consequently, this results in a reduction of $w$.

[19] 3:1 $\mathrm{H}_{2} \mathrm{SO}_{4}: 30 \% \mathrm{H}_{2} \mathrm{O}_{2}, 10$ min, $95{ }^{\circ} \mathrm{C} ; \mathrm{H}_{2} \mathrm{O}$ rinse, $10 \mathrm{~min} ; 1: 1: 40 \mathrm{NH}_{4} \mathrm{OH}: 30 \% \mathrm{H}_{2} \mathrm{O}_{2}: \mathrm{H}_{2} \mathrm{O}, 10$ min, $95{ }^{\circ} \mathrm{C} ; \mathrm{H}_{2} \mathrm{O}$ rinse, $10 \mathrm{~min} ; 1: 1049 \% \mathrm{HF}: \mathrm{H}_{2} \mathrm{O}, 10 \mathrm{~s} ; \mathrm{H}_{2} \mathrm{O}$ rinse, $10 \mathrm{~min}$.

[20]M. Born and E. Wolf, [Principles of Optics, $5^{\text {th }}$ Edition], Pergamon Press, Oxford, (1975).

[21]B. Carli and D. Iorio-Fili, "Absorption of composite bolometers," J. of the Optical Soc. of Am., 71, 1020, 1981.

[22]C. T. Black, K.W. Guarini, G. Breyta, M.C. Colburn, R. Ruiz, R. L. Sandstrom, E.M. Sikorski, and Y. Zhang, "Polymer self assembly in semiconductor microelectronics," J. Vac. Sci. Technol. B 24, 3188 (2006). 